\title{
Compton scattering sources and applications at LLNL
}

F. Albert, S. G. Anderson, G. Anderson, A. Bayramian, S. M. Betts, R. R. Cross, C. A. Ebbers, D. J. Gibson, R. A. Marsh, M. Messerly, M. Y. Shverdin, S. S. Wu, F. V. Hartemann, R. Scarpetti, C. W. Siders, C. P. Barty

\section{August 23, 2010}

Advanced accelerator concepts workshop Annapolis, MD, United States June 13, 2010 through June 19, 2010 
This document was prepared as an account of work sponsored by an agency of the United States government. Neither the United States government nor Lawrence Livermore National Security, LLC, nor any of their employees makes any warranty, expressed or implied, or assumes any legal liability or responsibility for the accuracy, completeness, or usefulness of any information, apparatus, product, or process disclosed, or represents that its use would not infringe privately owned rights. Reference herein to any specific commercial product, process, or service by trade name, trademark, manufacturer, or otherwise does not necessarily constitute or imply its endorsement, recommendation, or favoring by the United States government or Lawrence Livermore National Security, LLC. The views and opinions of authors expressed herein do not necessarily state or reflect those of the United States government or Lawrence Livermore National Security, LLC, and shall not be used for advertising or product endorsement purposes. 


\title{
Compton scattering sources and applications at LLNL
}

\author{
Felicie Albert, S.G. Anderson, G. Anderson, A. Bayramian, S.M. Betts, R.R. \\ Cross, C.A. Ebbers, D.J. Gibson, R.A. Marsh, M. Messerly, M.Y. Shverdin, S.S. \\ Wu, F.V. Hartemann, R. Scarpetti, C.W. Siders and C.P.J. Barty
}

Lawrence Livermore National Laboratory, NIF and Photon Science, 7000 east avenue, Livermore, CA, 94550

\begin{abstract}
We report the design and current status of a monoenergetic laser-based Compton scattering 0.5-2.5 MeV $\gamma$-ray source. Previous nuclear resonance fluorescence results and future linac and laser developments for the source are presented.
\end{abstract}

Keywords: Compton scattering, gamma-rays, X-band linac technology, nuclear photoscience.

PACS: $41.60 .-\mathrm{m}$, 07.85.Fv, 52.38. Ph

\section{INTRODUCTION}

At $\mathrm{MeV}$ photon energies relevant for nuclear processes, Compton scattering light sources are attractive because of their relative compactness and improved brightness above $100 \mathrm{keV}$, compared to typical $3^{\text {rd }}$ generation synchrotrons. Recent progress in accelerator physics and laser technology have enabled the development of a new class of tunable Mono-Energetic Gamma-Ray (MEGa-Ray) light sources based on Compton scattering between a high-brightness, relativistic electron beam and a high intensity laser pulse produced via chirped-pulse amplification (CPA). A new precision, tunable gamma-ray source driven by a compact, high-gradient X-band linac is currently under development and construction at LLNL. High-brightness, relativistic electron bunches produced by an X-band linac designed in collaboration with SLAC will interact with a Joule-class, $10 \mathrm{ps}$, diode-pumped CPA laser pulse to generate tunable $\gamma$-rays in the $0.5-2.5 \mathrm{MeV}$ photon energy range via Compton scattering. Based on the success of the previous Thomson-Radiated Extreme X-rays (T-REX) Compton scattering source at LLNL[1-3], the source will be used to excite nuclear resonance fluorescence lines in various isotopes; applications include homeland security, stockpile science and surveillance, nuclear fuel assay, and waste imaging and assay. After a brief presentation of successful nuclear resonance fluorescence (NRF) experiments done with T-REX, the new source design, key parameters, and current status are presented.

\section{NRF EXPERIMENTS}

T-REX experiments have been already performed on an enhanced version of the $100 \mathrm{MeV}$ LLNL electron linac used for previous Compton scattering sources. The electron bunch is accelerated to $\sim 120 \mathrm{MeV}$ and focused with a series of quadrupole magnets. The Interaction Laser System (ILS), that produces the photon beam to collide with the electrons, can deliver $750 \mathrm{~mJ}$ of energy in $25 \mathrm{ps}$ and $0.2 \mathrm{~nm}$ bandwidth at $10 \mathrm{~Hz}$ and $1064 \mathrm{~nm}$. The beam after the compressor can then be frequency doubled or tripled using large aperture DKDP crystals. The ILS is focused into a vacuum chamber, at $180^{\circ}$ with respect to the electron beam, by a $2.4 \mathrm{~m}$ focal length $\mathrm{f} / 50$ lens.

A complete spectral and spatial characterization of this Compton scattering light source has been made [3]: it produces photons with energies between $0.1 \mathrm{MeV}$ and $0.8 \mathrm{MeV}$. The T-REX source is described by its key parameters: size $\left(0.01 \mathrm{~mm}^{2}\right)$, divergence $\left(10 \times 6 \mathrm{mrad}^{2}\right)$, duration (ps), spectrum $(15 \%$ bandwidth $)$ and intensity $\left(10^{5}\right.$ photons/shot), all of which are summarized by an on-axis peak brightness of $2.5 \times 10^{15}$ photons $/ \mathrm{mm}^{2} / \mathrm{mrad}^{2} / \mathrm{s} / 0.1 \%$ 
bandwidth at $0.478 \mathrm{MeV}$. NRF measurements, a promising method for isotopic imaging, assay, and detection, were performed on ${ }^{7} \mathrm{Li}$ using this source [1].

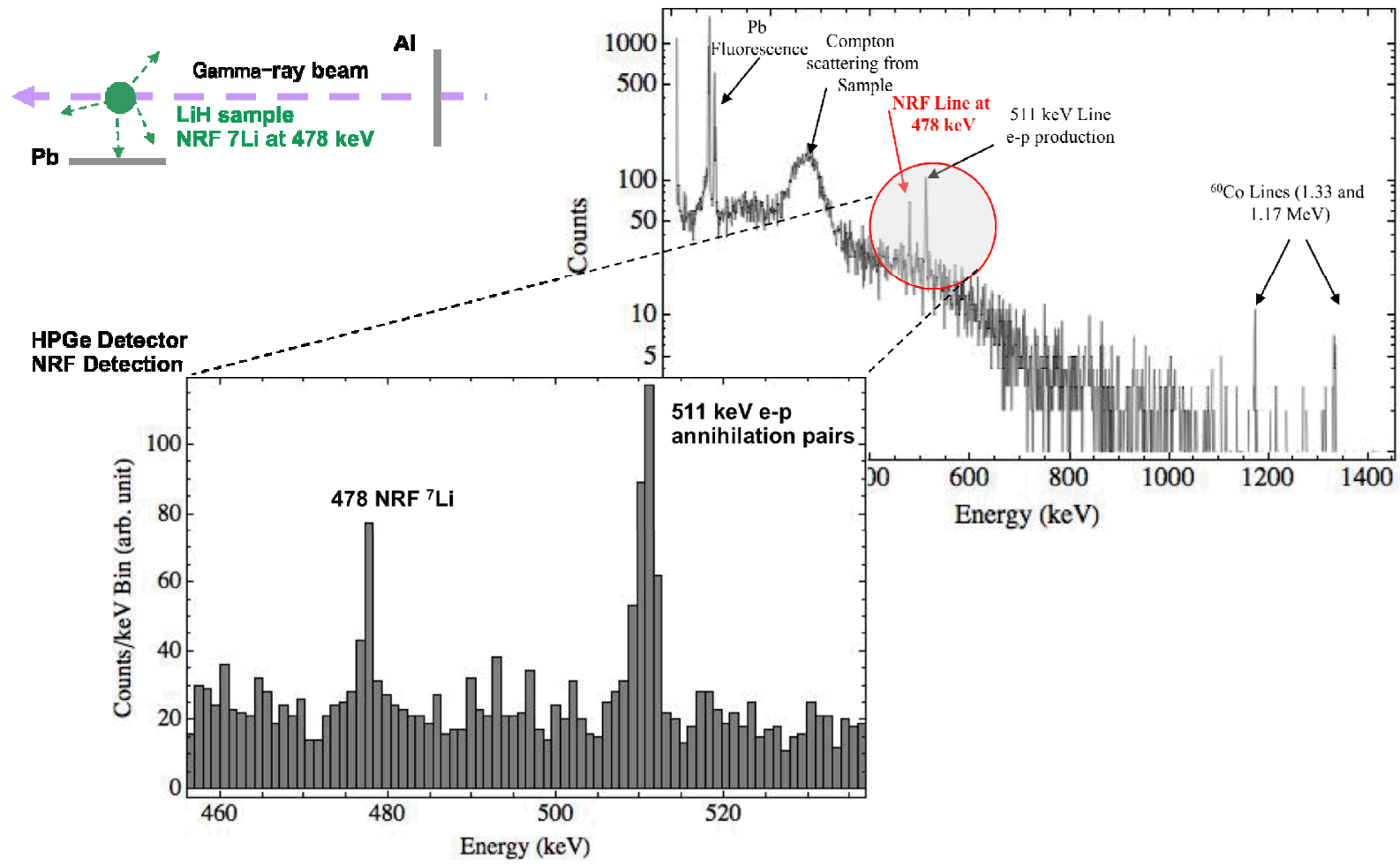

FIGURE 1. T-REX NRF results and detection of 7Li

\section{NEW SOURCE DESIGN}

Based on the successful results of T-REX, a new facility, shown in Figure 2, is currently under construction at LLNL, with the goal of generating tunable gamma-rays in the $0.5-2.5 \mathrm{MeV}$ photon energy range, at a repetition rate of $120 \mathrm{~Hz}$, and with a peak brightness in the $10^{19}$ photons/( $\left.\mathrm{s} \mathrm{x} \mathrm{mm^{2 }} \times \mathrm{mrad}^{2} \times 0.1 \% \mathrm{bw}\right)$.

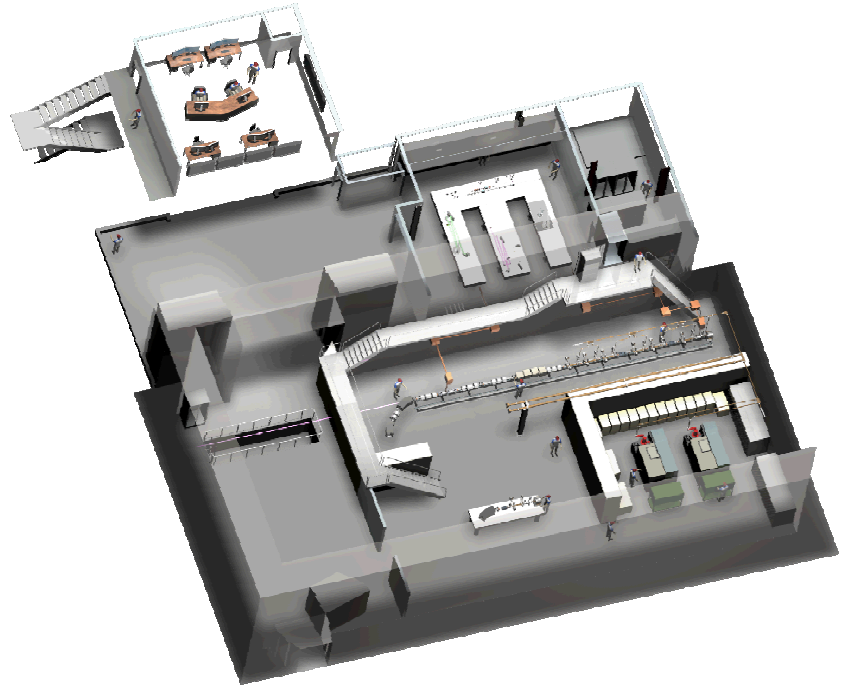

FIGURE 2. The new MEGa-ray facility 


\section{X-band rf photoinjector}

The rf photoinjector is based on an earlier high gradient $7 \mathrm{MeV}, 5.5$-cell X-band rf photoinjector [4]. Improvements specific to our application have been implemented and will be described in this paper. PARMELA simulations revealed that a longer first half- cell, as simulated with SUPERFISH resulted in a lower final emittance for the setup planned at LLNL. As a result a full redesign of the rf gun has been performed, using a longer first halfcell, lengthened from a 0.49 cell to a 0.59 cell. A schematic of the gun, low-energy beamline (LEB) and T53 is shown in Figure 3. Superior electron beam quality, with a normalized emittance of $0.4 \mathrm{~mm} . \mathrm{mrad}$ at $250 \mathrm{pC}$ charge, is ensured by the very high field applied to the photocathode: $200 \mathrm{MV} / \mathrm{m}$, nominally. Full emittance compensation is implemented, with an optimum distance from the photocathode to the first accelerating section of $0.8 \mathrm{~m}$.

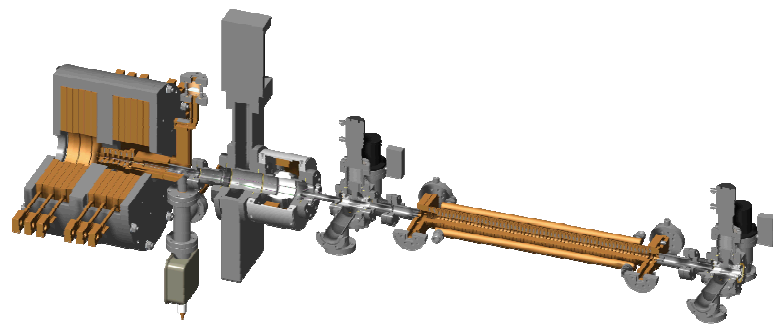

FIGURE 3. X-band rf gun, LEB, and T53 section.

The rf gun properties required for complete design are: field balanced across all cells, mode frequency of 11.424 $\mathrm{GHz}$, and a coupling $\beta$ of $\sim 1.7$. This new rf gun boasts an improved mode separation of $>20 \mathrm{MHz}$, which decreases mode beating of the electric field on the cathode. The new rf gun also employs a racetrack coupler to reduce the rf quadrupole field experienced by the electron beam, and elliptically contoured irises to decrease the maximum surface electric field. These improvements were incorporated into the design of a modified rf gun for LLNL.

Complete 3D rf design for the photoinjector was accomplished using HFSS. Each modification affects the three design criteria: field balance is primarily a function of relative cell radii; coupling is primarily a function of the coupler cell radius and coupling aperture; the frequency is primarily changed by scaling all cell radii. Each adjustment changes the primary goal being modified, but also affects the other two. Final design is achieved by successive iteration, until all parameters are simultaneously met. The final field balance is quite excellent, and the final coupling was achieved at $11.424 \mathrm{GHz}$, with a $\beta$ of $\sim 1.7$.

Final modification of the design is necessary to converge on a set of dimensions for engineering drawings and actual OFHC Cu fabrication. Machining will be done at $20^{\circ} \mathrm{C}$, while operation is planned for $45^{\circ} \mathrm{C}$. Scaling of the design dimensions was calculated and simulated. Design numbers were then truncated to acceptable fabrication tolerances, which required readjustment of the drawing numbers to conform with optimal field balance, coupling, and frequency at the operating temperature of $45^{\circ} \mathrm{C}$. Engineering drawings have been completed, and fabrication is planned in the near future.

\subsection{GW X-band rf system}

The high power $\mathrm{rf}$ system is to provide adequate rf power to the accelerator to achieve the end-point electron energy. The accelerator consists of a X-band photo-gun and six sections of traveling wave accelerating structure, the T53VG3. The X-band photo-gun is a modified version of the 5.49 cells rf gun tested at SLAC in 2002. The rf budget for the gun is $20 \mathrm{MW}$ and the fill time of the structure is $65 \mathrm{~ns}$. The T53VG3 type travelling wave structure was extensively tested for high gradient operation and has operated at high gradient with low breakdown rates [5]. The T-series structures are essentially the low group velocity (downstream) portion of the original $1.8 \mathrm{~m}$ structures [6]. This structure can be operated with acceptable trip rate at gradients up to $90 \mathrm{MV} / \mathrm{m}$. The fill time of this structure is $74.3 \mathrm{~ns}$ and an rf power of $70 \mathrm{MW}$ is budgeted for each section.

The high power rf source is a X-band klystron (XL-4), which was developed by SLAC in the mid 90's for the high power testing of the X-band structures. The XL-4 is a solenoid focused klystron which requires a 0.47 Tesla 
solenoid. The high voltage pulse required by the klystron is provided by a state of the art, solid-state high voltage modulator. We have chosen the solid-state modulator (K2-3X) built by ScandiNova for its pulse-to-pulse stability and solid-state modular design; the first of these modulators has been delivered and is awaiting installation. Two klystrons and two high voltage modulators are planned for the LLNL MEGa-Ray project.

The high power pulsed rf output of two klystrons is $100 \mathrm{MW}, 1.5 \mathrm{msec}$. The high power rf needs of the rf gun and accelerator sections are $440 \mathrm{MW}$ for $210 \mathrm{~ns}$ ( $\sim 3 \mathrm{x}$ fill time). The logical way to achieve this is to pulse compress the output of the klystrons to $500 \mathrm{MW}, 210 \mathrm{~ns}$ and to distribute the compressed pulse to the rf gun and accelerating sections. SLAC has developed and demonstrated SLED II with multi-mode delayed lines with similar power gain factor [7]. The dual-mode SLED-II delay lines will be approximately 15 meter long with inner diameter of $17 \mathrm{~cm}$.

$500 \mathrm{MW}, 210 \mathrm{~ns}$ pulses are the desired output of the pulse compression system. These compressed pulses will be fed into a $13 \mathrm{~dB}$ coupler. The $13 \mathrm{~dB}(25 \mathrm{MW})$ output will go to the rf gun. To allow for tuning and control, a phase shifter and attenuator are put in this arm. A barrier window is also planned for the rf gun. This is to limit the number of times the rf gun is exposed to air and to possibly provide for a configuration in which the rf gun can be baked and sealed as a unit before installation. The rest of the compressed power $(475 \mathrm{MW})$ is to be distributed to the linear accelerator sections. A $3 \mathrm{~dB}$ H-hybrid is used to divide the $475 \mathrm{MW}$ in half. Then a combination and $4.8 \mathrm{~dB}$ and 3 $\mathrm{dB}$ H-hybrids are used to distribute the power in one third portion $(\sim 70 \mathrm{MW})$ to each T53 section. Phase shifters and other control elements will be added as needed.

\section{Synchronization}

To ensure synchronization between the electron-generating laser pulse, the scattering laser pulse, and the electronaccelerating rf power, the fiber-based laser oscillator is used as a universal clock. The oscillator runs at a subharmonic of the rf design frequency ( $40.8 \mathrm{MHz}$ for a $11.424 \mathrm{GHz}$ accelerator), and provides seed light to both amplifier systems. The oscillator pulse train is monitored by a high-speed photodiode and the $11.424 \mathrm{GHz}$ component of the signal is selected by a narrow band-pass filter, amplified, and delivered to the rf power system. Because the laser pulses start from the same oscillator, they are guaranteed to have good relative stability - all that is required it to match the travel time to the interaction point. Selecting correct pulses from the oscillator will get the pulses within $24 \mathrm{~ns}$, and an extra length of optical fiber in the pre-amplification system can get to $\sim \mathrm{ns}$ resolution. Final ps accuracy comes from an optical delay arm with micron accuracy.

\section{Photocathode drive laser}

The photo-electrons are generated by a $50 \mu \mathrm{J}, 263 \mathrm{~nm}$ spatially and temporally shaped laser pulse. The oscillator seed is amplified (using standard chirped-pulse amplification [CPA] methods) in a series of Yb-doped fiber amplifiers, beginning with standard 6- $\mu \mathrm{m}$ core pre-amps, a $29 \mu \mathrm{m}$ core intermediate photonic crystal fiber stage and a final $85 \mu \mathrm{m}$ fiber rod amplifier to generate $1 \mathrm{~mJ}, 1053 \mathrm{~nm}$ pulses at $120 \mathrm{~Hz}$ that are compressed to $250 \mathrm{fs}$. These pulses are frequency quadrupled, stacked in a "Hyper-Michelson" pulse stacker, which converts the single input pulse into 8 replicas using 3 beam splitters; then transformed from Gaussian to a flat-top transverse profile using refractive optical beam shaping components. This beam is transported to the photoinjector, resized to $1 \mathrm{~mm}$, and imaged onto the cathode surface.

\section{Interaction laser system}

Because the scattering laser should have minimal bandwidth and needs to be no shorter than $10 \mathrm{ps,} \mathrm{Nd:YAG} \mathrm{with}$ its narrow gain bandwidth is a suitable material for amplification. Because of the narrow $(<1 \mathrm{~nm})$ bandwidth and long $(>\mathrm{ns})$ desired stretched pulse length in the amplification chain, we developed a novel hyper-dispersion stretcher and compressor pair [8] that provides very high ( $>7000 \mathrm{ps} / \mathrm{nm})$ dispersion. The scattering laser pulse is generated by pre-amplifying a selected oscillator pulse in a $6 \mu \mathrm{m}$ and $29 \mu \mathrm{m}$ core fibers to the $30 \mu \mathrm{J}$ level, then 
doing final amplification in a set of diode-pumped Nd:YAG heads. The diode-pumping architecture allows this system to produce $1 \mathrm{~J}$ laser pulses at $120 \mathrm{~Hz}$; subsequently compressed to $10 \mathrm{ps}$. This beam is then frequencydoubled to $532 \mathrm{~nm}$ and transported to the interaction region.

\section{Interaction region}

For the interaction of the laser and electron beams, a $180^{\circ}$ geometry was chosen to maximize the gamma flux and minimize temporal tolerance. The electron beam is focused by a quadrupole triplet and re-collimated after the interaction by a matching triplet. The laser beam is focused by a $70 \mathrm{~cm}$ focal length lens, and steered onto the electron beam path by a dielectrically coated mirror with a hole in the center (which allows the electron beam to propagate through). The beam is collected by a similar mirror, on the other side of the interaction. To establish the relative alignment of the laser and electron beams, an optically polished nickel cube is located at the interaction point. Oriented with the vertical faces at $45^{\circ}$ to the electron-laser beamline, the (significantly attenuated) laser reflects off the surface and the electron beam generates optical transition radiation light. These beams are imaged with a camera to ensure they are aligned vertically, and the overlap horizontally can be determined by seeing the beams meet at the edge. The light is also imaged onto a streak camera to allow the adjustment of the laser delay to get the correct temporal overlap.

\section{Expected gamma-ray performance}

Different benchmarked codes (with T-REX) have been used to predict the gamma-ray output of the new source. In this case, the dose is expected to be $|x|$; the electron bunch duration $2 \mathrm{ps}$, the electron beam energy $250 \mathrm{MeV}$, and its normalized emittance $1.5 \mathrm{~mm}$.mrad. This translates into a peak brightness of order $10^{19}$ photons $/\left(\mathrm{s} \mathrm{x} \mathrm{mm}^{2} \mathrm{x}\right.$ $\left.\operatorname{mrad}^{2} \times 0.1 \% \mathrm{bw}\right)$. More accurate estimates yield projected brightness numbers ranging between $10^{18}$ and $10^{20}$, in the same units. These models use the Compton formula, which includes recoil, and the Lorentz-boosted Klein-Nishina differential scattering cross-section; they also provide estimates of the source linewidth, which is bounded by the laser spectrum and relative energy spread of the electron beam, on the high-frequency side, and by the electron beam emittance in the low-energy tail due to electrons crossing the focus at off-axis angles. Nonlinear spectral broadening effects have also been studied for the design [9]. This very narrow bandwidth will enable the detailed NRF applications outlined in the introduction.

\section{ACKNOWLEDGMENTS}

This work performed under the auspices of the U.S. Department of Energy by Lawrence Livermore National Laboratory under Contract DE-AC52-07NA27344.

\section{REFERENCES}

1. F. Albert, S.G. Anderson, G.A. Anderson, S.M. Betts, D.J. Gibson, C.A. Hagmann, J. Hall, M.S. Johnson, M.J. Messerly, V.A. Semenov, M.Y. Shverdin, A.M. Tremaine, F.V. Hartemann, C.W. Siders, D.P. McNabb and C.P.J. Barty, Opt. Letters, 35, vol. 3 354-357 (2010).

2. D.J. Gibson, F. Albert, S. G. Anderson, S. M. Betts, M. J. Messerly, H. H. Phan, V. A. Semenov, M. Y. Shverdin, A. M. Tremaine, F. V. Hartemann, C. W. Siders, D. P. McNabb, and C. P. J. Barty, Phys. Rev. ST accel. Beams, 070703 (2010).

3 F. Albert, S. G. Anderson, D. J. Gibson, C. A. Hagmann, M. S. Johnson, M. Messerly, V. Semenov, M. Y. Shverdin, B. Rusnak, A. M. Tremaine, F. V. Hartemann, C. W. Siders, D. P. McNabb, and C. P. J. Barty, Phys. Rev. ST accel. Beams, 070704 (2010).

4 A. Vlieks, et al., in High Energy Density and High Power RF: $5^{\text {th }}$ Workshop, AIP CP625, p. 107 (2002); R.A. Marsh, et al. "Advanced X-Band Test Accelerator for High Brightness Electron and Gamma Ray Beams", IPAC2010, Kyoto, Japan.

5 C. Adolphsen, et al. "Normal-Conducting RF Structure Test Facilities and Results", PAC2003, Portland USA.

6 G. Caryotakis, "The X-Band Klystron Program at SLAC", SLAC-PUB- 7146, April, 1996 
7 S. G. Tantawi, et al. "High-power multimode $X$-band rf pulse compression system for future linear colliders", Phy. Rev. Special Topics - Accel. \& Beams 8, 042002 (2005).

8 M. Y. Shverdin, F. Albert, S. G. Anderson, S. M. Betts, D. J. Gibson, M. J. Messerly, F. V. Hartemann, C. W. Siders, and C. P. J. Barty, Opt. Lett, 35, 14 (2010).

9 F.V. Hartemann, F. Albert, C.W. Siders and C.P.J. Barty, Phys. Rev. Lett., In press (2010). 